\title{
Atomistic simulations of grain boundary migration under recrystallisation conditions
}

DOI:

10.1088/1361-651X/ab2176

\section{Document Version}

Accepted author manuscript

Link to publication record in Manchester Research Explorer

\section{Citation for published version (APA):}

Race, C. (2019). Atomistic simulations of grain boundary migration under recrystallisation conditions. Journal of Modelling and Simulation in Materials Science and Engineering. https://doi.org/10.1088/1361-651X/ab2176

\section{Published in:}

Journal of Modelling and Simulation in Materials Science and Engineering

\section{Citing this paper}

Please note that where the full-text provided on Manchester Research Explorer is the Author Accepted Manuscript or Proof version this may differ from the final Published version. If citing, it is advised that you check and use the publisher's definitive version.

\section{General rights}

Copyright and moral rights for the publications made accessible in the Research Explorer are retained by the authors and/or other copyright owners and it is a condition of accessing publications that users recognise and abide by the legal requirements associated with these rights.

\section{Takedown policy}

If you believe that this document breaches copyright please refer to the University of Manchester's Takedown Procedures [http://man.ac.uk/04Y6Bo] or contact uml.scholarlycommunications@manchester.ac.uk providing relevant details, so we can investigate your claim.

\section{OPEN ACCESS}




\title{
Atomistic simulations of grain boundary migration under recrystallisation conditions
}

\author{
C P Race ${ }^{1}$ \\ ${ }^{1}$ School of Materials, University of Manchester, Manchester, M13 9PL, United \\ Kingdom \\ E-mail: christopher.race@manchester.ac.uk
}

\begin{abstract}
We present details of an approach to simulating the migration of grain boundaries using classical molecular dynamics in a regime in which the boundary motion is driven by the presence of dislocation defects resulting from plastic deformation. By simulating the shrinkage of spherical grains of deformed material embedded in a perfect crystal, we create conditions in which grain boundary motion takes place on time scales accessible to molecular dynamics simulations, in which the defect free energy acts as a driving force additional to the capillary force due to excess grain boundary energy. This approach is particularly flexible in allowing arbitrary choices of misorientation axis and angle and providing an interface in which all grain boundary plane orientations are represented. This flexibility is at the cost of additional complexity in the system under study and we establish an approach suitable for analysing the excess grain boundary energy and the components of the force for boundary motion on a local basis over the surface of the shrinking grain. We demonstrate that this approach is able to resolve variation in the grain boundary energy related to the intrinsic grain boundary dislocation network in lower angle boundaries and to detect local maxima in the driving force due to the dislocation structure within the deformed grain. We show that the variation in measured grain boundary velocities is accounted for by the excess energy of the dislocations. We further show that the presence of dislocations in the shrinking grain has a tendency to reduce the anisotropy in grain boundary behaviour. Indicative evidence is presented that the dislocation network may act as a focal point for mechanisms of grain boundary migration.
\end{abstract}

\section{Introduction}

The motion of grain boundaries in polycrystalline metals undergoing primary recrystallisation is critically important in establishing the grain structures responsible for the properties that those metals require in application. This grain boundary motion is driven by an asymmetry in the free energy between the two grains either side of the boundary: pristine grains grow at the expense of material permeated by a network of dislocations introduced via mechanical processing Humphreys and Hatherly 2004. The presence of a high density of defects in the vicinity of the grain boundary means that boundary motion may proceed in a different manner and via very different mechanisms to motion of grain boundaries in the otherwise defect free polycrystals and bicrystals that are more frequently the objects of study Homer 2015. The properties of the grain boundaries might be altered significantly, with, for example, a reduction in variability between boundaries or in anisotropy with boundary plane. Furthermore, the excess free energy embodied in dislocation networks 
is amongst the strongest thermodynamic driving forces for grain boundary migration encountered in real materials Sutton and Balluffi 1995, Gottstein and Shvindlerman 2010, reaching forces of up to $\sim 100 \mathrm{MPa}$, which raises the possibility of directly simulating the process of grain boundary migration using molecular dynamics (MD) under realistic conditions. This is in contrast to the anomalously high forces, often artificial in nature, that must be applied to drive boundaries in simulations of pristine material.

Most simulations of the process of recrystallisation are performed at the mesoscale Miodownik 2002. Such approaches are very useful for exploring the phenomenology of recrystallisation, but rely on an explicit encoding of the known physics of the process into the simulation methodology. Where aspects of the physics are unknown, for example details of the underlying atomic-scale processes, then key features may be absent in the mesoscale models and their effects only 'corrected for' in adjustable parameters of the model in a potentially unphysical manner. It is therefore important to complement mesoscale simulations with atomic scale studies in which details of the physics can emerge.

To date, very little atomistic simulation work has been performed to address this scenario. Godiksen et al.Godiksen et al. 2005] proposed a geometry for a bicrystal molecular dynamics simulation cell in which the motion of a pair of boundaries was driven by a second set of grain boundaries permeating though the central grain and perpendicular to the boundaries whose motion was to be studied. They employed this set up in a series of studies Godiksen et al. 2007a b, 2008 exploring the effect of different types of grain boundary networks within the central crystal, of the strength of the driving force, of temperature and of the empirical potential used in the simulation.

The work of Godiksen et al. makes use of a carefully controlled geometry in which the bicrystal boundaries under study and the dislocation network used to drive their motion are well defined. This allows for systematic study of the behaviour of the system as conditions are varied, but does come with some drawbacks. First, the choice of an overall bicrystal geometry and of a structure of planar dislocation arrays in the central grain restricts the geometry of the grain boundaries that can be studied and the nature of the dislocations between them. Second, whilst dislocation cell structures are a prominent feature of deformed fcc metals Driver et al. 1994, they are not the only one. In particular, localised regions of very high dislocation density are observed in some grains in severely deformed aluminium Liu et al. 2008. The work presented here is in the spirit of Ref Godiksen et al. [2005], which provided a preliminary demonstration of the plausibility of the bicrystal method of Godiksen et al., but demonstrating a complementary approach. Rather than use a carefully controlled simulation setup, we instead aim for a method in which the motion of a general grain boundary is driven by a dense network of dislocations.

In this paper, we present the results of simulations that demonstrate one possible approach to simulating the motion of a grain boundary under the conditions prevailing during primary recrystallisation. We first outline the details of the method, before presenting and discussing some preliminary results. Finally, we offer conclusions and discuss useful next steps for applying the approach to systematically explore defectdriven grain boundary motion. 


\section{Methods}

\subsection{Simulating boundary migration}

In order to observe the effect of a network of dislocation defects on grain boundary migration and the mechanisms involved we need a simulation methodology which gives rise to grain boundary migration within a timeframe achievable within molecular dynamics and in which the grain boundary motion is, at least in part, driven by the presence of the defects. Simulations of bicrystals have great appeal and are often used for systematic studies of boundary motion, because they isolate the behaviour of a well defined boundary within the five-dimensional parameter space of the macroscopic degrees of freedom of grain boundary geometry. Our early experiments with this geometry, involving a slab of plastically deformed material sandwiched between pristine grains (see Figure 1(a)) were not promising. The dislocation network led to apparent immobilisation of a boundary otherwise known to be mobile Race et al. 2014 and the presence of the boundary encouraged rapid recovery of the dislocation network, with the defects being absorbed by the interfaces. A tendency for grain boundaries to absorb dislocations without moving was also observed by Godiksen et al Godiksen et al. 2007a. An alternative approach would place a spherical grain of deformed material in a perfect bulk cell. This sacrifices the simplicity of the bicrystal and leaves us with a grain boundary whose interfacial normal varies over its surface, but does relax the restrictions that periodic boundaries impose on the geometries that can be simulated, permitting arbitrary choices for misorientation axis and angle.
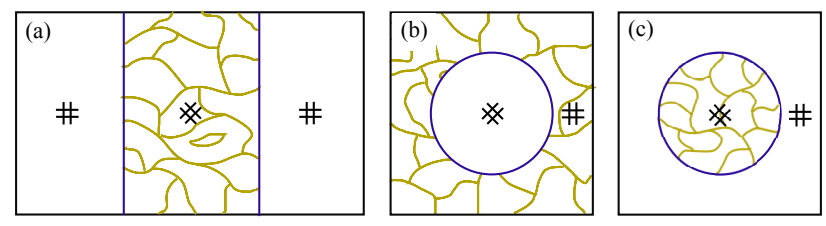

Figure 1. Schematic diagrams of possible approaches to simulating dislocationdriven grain boundary motion. Grain boundaries are shown in blue and dislocation networks by yellow lines. (a) a bicrystal cell; (b) a spherical inclusion with a configuration analogous to the growth of perfect crystal grains in deformed material; (c) the "inside-out" configuration used in the present work. The \# symbols are a schematic indication of local crystal orientation to emphasise the misorientation between different regions of the simulation cell.

The supercell construction that would most closely resemble the growth of grains in primary recrystallisation is one in which we insert a spherical grain of perfect crystal into a defect-laden matrix grain, as shown in Figure 1(b). However, in this scenario the driving force due to the dislocation defects must be strong enough to overcome the capillary force if the grain is not to shrink. Even for a very high dislocation density of $10^{17} \mathrm{~m}^{-2}$ the driving pressure will only amount to $\sim \mathrm{meV} \AA^{-3}$. If we assume a representative energy density for the grain boundary of $\gamma \sim \mathrm{eV} \AA^{-2}\left(\sim 0.1 \mathrm{Jm}^{-2}\right)$ then this would require a grain of radius at least $\sim 100 \mathrm{~nm}$, if the defect driving force were to dominate. This in turn requires a simulation cell containing $\sim 100$ million atoms. Whilst this is an achievable length scale for MD, such simulations would be very costly and preclude much exploration of the system behaviour over parameter space. 
Instead we choose to simulate an "inside-out" scenario of a misoriented spherical inclusion of defect-laden material in a perfect crystal, in which both the capillary driving force due to the grain boundary and the driving force due to the dislocation defects act to shrink the central grain. This guarantees that the grain boundary will move on MD timescales at the system sizes and dislocation densities that we are able to study. We construct cubic supercells of perfect crystal of $\sim 20 \mathrm{~nm}$ across, containing around 0.5 million atoms, in which a central sphere of radius $6 \mathrm{~nm}$ is removed and replaced with the same volume of plastically deformed material. This central sphere is rotated about a chosen misorientation axis by a given misorientation angle to create a spherical grain boundary. To calculate interatomic forces we use an existing EAM potential for aluminium Zope and Mishin 2003] which we have previously verified as suitable for simulating grain boundary migration in bicrystals Race et al. 2014] and shown to reproduce at least one energy barrier associated with atomic rearrangement during grain boundary migration Race et al. 2015. We never the less make no claims to be studying aluminium specifically and instead employ this potential as a reasonable model of interatomic forces in a model metal and without any known pathologies. All our simulations are carried out in Lammps Plimpton [1995] with fully periodic boundary conditions, with a Nose-Hoover thermostat applied to maintain a constant temperature and a zero-pressure barostat.

We emphasise that the approach we take here is far from a realistic representation of typical recrystallistion conditions. We simulate only a single, spherical deformed grain, within a single defect-free surrounding grain. More realistic conditions would involve non-spherical grains, a more complex microstucture of the surrounding material and (as remarked above) growth, rather than shrinkage, of the defect-free region. As we will see in Section 3.1, we also simulate an atypical deformation structure, with much higher dislocation densities and a different morphology to those typically encountered in recrystallisation. Rather than begin immediately with more realistic simulations, our aim here is to develop an approach to simulation in which grain boundary motion is driven by the stored energy of plastic deformation and to demonstrate a method for analysing such simulations.

\subsection{Obtaining deformed material}

In order to create simulation supercells with our chosen geometry we first need a library of samples of material containing dislocation networks with a range of dislocation densities. To obtain such samples we follow a similar procedure to that used by Zepeda-Ruiz et al Zepeda-Ruiz et al. [2017]. We take a cubic block of 500,000 atoms, with fully periodic boundary conditions and we insert a population of six $1 / 2\langle 110\rangle$-type dislocation loops of $12 \AA$ radius at random locations, to act as nuclei for dislocation production. We thermalise the cell to a temperature of $300 \mathrm{~K}$, and then compress it uniaxially by some target strain between $20 \%$ and $98 \%$ over the course of $200 \mathrm{ps}$ (200k timesteps of $1 \mathrm{fs}$ ). We then apply a biaxial compression along the other two orthogonal directions to return the cube to its original dimensions, again over the course of $200 \mathrm{ps}$. The timescales used for the deformation in our simulations imply very high strain rates: for the initial uniaxial compression the strain rate is in the range $2 \times 10^{8} \mathrm{~s}^{-1}$ to $6.4 \times 10^{9} \mathrm{~s}^{-1}$, orders of magnitude beyond experimental strain rates. However, we are working with a very small sample (only $20 \mathrm{~nm}$ across), and from an atomic point of view, the typical atomic velocity implied by the strain rate is only $\sim 10 \mathrm{~m} \mathrm{~s}^{-1}$, which corresponds to a high, but not ultra-high, strain rate 
experiment on a macroscopic sample.

\subsection{Analysing a spherical grain}

Here we discuss our approach to calculating the driving forces associated with grain boundary migration during our simulations. Because we are simulating a spherical grain, the grain boundary plane, and therefore the interfacial energy, will vary over the surface of the spherical inclusion. The introduction of a dislocation network in the central grain adds further complexity as the excess energy associated with these dislocations will be localised around the dislocation lines. We therefore adopt an approach to calculating components of the driving force locally over the surface of the grain. We treat the system as fundamentally atomistic and so the driving forces are assumed to arise from excess energies of the atoms in a given region, either as a result of the interface itself or of the dislocation network in the deformed central grain. We will present the basis of this analysis by considering an idealised model system and later demonstrate its function on the messy, dynamic system in Section 3 .

We consider a portion of our system lying between polar angles $\theta$ and $\theta+\mathrm{d} \theta$ and between azimuthal angles $\phi$ and $\phi+\mathrm{d} \phi$ (see Figure 2(a)). What we wish to do is calculate the excess energy $\Delta E$ of this fraction of the sphere as the sum of contributions $\Delta E_{\mathrm{Dis}}$ and $\Delta E_{\mathrm{GB}}$ due to the plastic deformation and grain boundary respectively. In the following discussion we will assume that in our idealised system the mean excess potential energy per atom $\varepsilon$ (compared with the energy per atom in the perfect bulk) varies smoothly as a function of distance $\rho$ from the centre of the grain as shown in Figure 2(b). This assumption will be relaxed in the final, atomistically-based, implementation. We consider $\varepsilon$ to be made up of two contributions:

$$
\begin{aligned}
\varepsilon_{\text {Disl }}(\rho ; \theta, \phi) & =\Delta \varepsilon & & \rho \leq r \\
& =0 & & \rho>r,
\end{aligned}
$$

due to the dislocation network and

$$
\begin{aligned}
\varepsilon_{\mathrm{GB}}(\rho ; \theta, \phi) & >0 & & r-w<\rho<r+w \\
& =0 & & \rho<r-w \quad \text { or } \quad \rho>r+w,
\end{aligned}
$$

due to the grain boundary, which is assumed to have a nominal width of $2 w . r$ is the radius of the spherical inclusion at this point on the surface. This case is illustrated schematically in Figure 2 (b).

We would like to be able to write the excess energy of this angular portion of our system as a sum of dislocation and grain boundary contributions, $\Delta E=$ $\Delta E_{\mathrm{Disl}}+\Delta E_{\mathrm{GB}}$, where

$$
\Delta E_{\text {Disl }}=\int_{0}^{r} \mathrm{~d} \rho \mathrm{d} \Omega \rho^{2} n \varepsilon_{\text {Disl }}
$$

and

$$
\Delta E_{\mathrm{GB}}=\int_{r-w}^{r+w} \mathrm{~d} \rho \mathrm{d} \Omega \rho^{2} n \varepsilon_{\mathrm{GB}},
$$

where $\mathrm{d} \Omega=\sin \theta \mathrm{d} \theta \mathrm{d} \phi$ is the solid angle element defined by the angular intervals $\mathrm{d} \theta$ and $\mathrm{d} \phi$ around $\theta$ and $\phi . \quad n$ is the number density of atoms, which we assume to be constant for the sake of simplicity, again relaxing this assumption in the final

implementation, 
The driving force for grain boundary migration, written as a pressure, is given by

$$
P=P_{\text {Disl }}+P_{\mathrm{GB}}=\frac{1}{r^{2} \mathrm{~d} \Omega} \frac{\mathrm{d}}{\mathrm{d} r}\left(\Delta E_{\text {Disl }}+\Delta E_{\mathrm{GB}}\right),
$$

where $r^{2} \mathrm{~d} \Omega$ is the surface area of the interface in the portion of the system within solid angle $\mathrm{d} \Omega$.

We can confirm that these contributions have the expected form by writing the grain boundary energy density

$$
\gamma=\frac{1}{r^{2} \mathrm{~d} \Omega} \int_{r-w}^{r+w} \mathrm{~d} \rho \mathrm{d} \Omega \rho^{2} n \varepsilon_{\mathrm{GB}}
$$

Then we have

$$
P_{\text {Disl }}=n \Delta \varepsilon,
$$

an excess energy density due to the dislocations, and

$$
P_{\mathrm{GB}}=\frac{2 \gamma}{r},
$$

the expected capillary force.

For clarity, we note that because we are assuming that the only change occurring to a segment of grain boundary as it migrates is to its area, rather than also its inclination, we are implicitly neglecting the role of grain boundary stiffness in providing a driving force. This stiffness component of the force can be significant in magnitude and show strong anisotropy Abdeljawad et al. [2018], but we believe we are justified in neglecting it here because we focus on the early stages of grain shrinkage, in which the grain remains close to spherical and there is therefore negligible rotation of the grain boundary plane segments during migration. Furthermore, attempting to measure the local grain boundary stiffness on an equivalent basis to our atomistic calculation of the grain boundary energy and dislocation contributions to the driving force would be complex and lies outside the scope of the current work.

Now, in dealing with our atomistic system we will have only the total potential energy of each atom to work with and so the excess energy of our system will need to be partitioned somewhat differently as $\Delta E=\Delta E_{\text {Inner }}+\Delta E_{\text {Interface, }}$, where,

$$
\Delta E_{\text {Inner }}=\int_{0}^{r-w} \mathrm{~d} \rho \mathrm{d} \Omega \rho^{2} n \varepsilon_{\text {Disl }}
$$

and

$$
\Delta E_{\text {Interface }}=\int_{r-w}^{r+w} \mathrm{~d} \rho \mathrm{d} \Omega \rho^{2} n\left(\varepsilon_{\mathrm{GB}}+\varepsilon_{\text {Disl }}\right) .
$$

We can calculate the driving forces $P_{\text {Inner }}$ and $P_{\text {Interface }}$ corresponding to these excess energy contributions, but these must then be corrected to obtain the contributions of interest:

$$
P_{\text {Disl }}=P_{\text {Inner }}+\Delta_{P}
$$

and

$$
P_{\mathrm{GB}}=P_{\text {Interface }}-\Delta_{P},
$$

where

$$
\Delta_{P}=\frac{1}{r^{2} \mathrm{~d} \Omega} \frac{\mathrm{d}}{\mathrm{d} r} \int_{r-w}^{r} \mathrm{~d} \rho \mathrm{d} \Omega \rho^{2} n \varepsilon_{\text {Disl }}
$$


is the necessary correction. Since $\varepsilon_{\text {Disl }}=\Delta \varepsilon$, this leads to

$$
\Delta_{P}=n \Delta \varepsilon \frac{w(2 w-r)}{r^{2}} .
$$

This term corrects for a misattribution of part of the excess potential energy due to the dislocation network in the spherical inclusion as a contribution to the interfacial energy.

The purpose of the above discussion was to demonstrate the correspondence between excess atomic potential energies in different regions and the two contributions to grain boundary driving force, and to derive a form for the correction term $\Delta_{P}$. We will see in Section 3.2 .2 how this correction is required for a correct interpretation of the dynamical simulations. We now describe how we implement the above approach to analysis in the case of our atomistic simulation cells.

The starting point is a set of positions $\{\mathbf{R}\}$ and potential energies $\{u\}$ for the atoms at a given time in our simulations. To simplify the analysis the atomic configuration is first fully relaxed, using a BFGS minimisation procedure, to remove thermal noise. For all the atoms lying within a solid angle element $\Delta \Omega$ defined by angular ranges $\Delta \theta$ and $\Delta \phi$ about the centre of the inclusion we then calculate the numbers of atoms $N_{i}$, volumes $V_{i}$ and total potential energies $E_{i}$ for the atoms lying in three intervals of radial distance $\rho$ from the centre of the inclusion. These regions are labelled by $i \in\{$ Inner, GB, Bulk $\}$ as indicated in Figure 2(c). The 'Inner' and 'Bulk' regions have width $d$, and the 'GB' region has width $w$. The grain boundary region spanf the nominal location of the grain boundary, which is first found by examining the average value of the grain boundary order parameter introduced by Janssens et al.Janssens et al. 2006 and implemented in the Lammps code Plimpton 1995]. We can then calculate a measure of the local grain boundary energy density

$$
\gamma(\theta, \phi)=\frac{E_{\mathrm{GB}}-\left(N_{\mathrm{GB}} / N_{\mathrm{Bulk}}\right) E_{\mathrm{Bulk}}}{r^{2} \sin \theta \Delta \theta \Delta \phi},
$$

and pressure due to the dislocations in the region inside the boundary,

$$
P_{\text {Disl }}(\theta, \phi)=\frac{E_{\text {Bulk }}}{V_{\text {Bulk }}}-\frac{E_{\text {Inner }}}{V_{\text {Inner }}}+\Delta_{P}
$$

where we approximate the correction term as

$$
\Delta_{P}=n \Delta \varepsilon \frac{w(2 w-r)}{r^{2}}=\frac{E_{\text {Inner }}}{V_{\text {Inner }}} \frac{w(2 w-r)}{r^{2}} .
$$

\section{Results and discussion}

\subsection{Deformation simulations}

Before discussing the results of our simulations of grain boundary migration, we first consider the deformed material used to construct the supercells with strained spherical inclusions. This is obtained from the simulations described in Section 2.2. Figure 3 shows the dislocation structures for each of the six chosen strains following the biaxial compression to return the cell to a cubic shape. Quantitative data for these dislocation networks is given in Table 3.1 and we can see that the total line length varies approximately linearly with the plastic strain (Figure 4). For all but the largest

‡ The 'GB' region is offset somewhat so that $75 \%$ of its width lies inside the grain boundary as this was found to better match the region of excess atomic potential energy associated with the interface. 


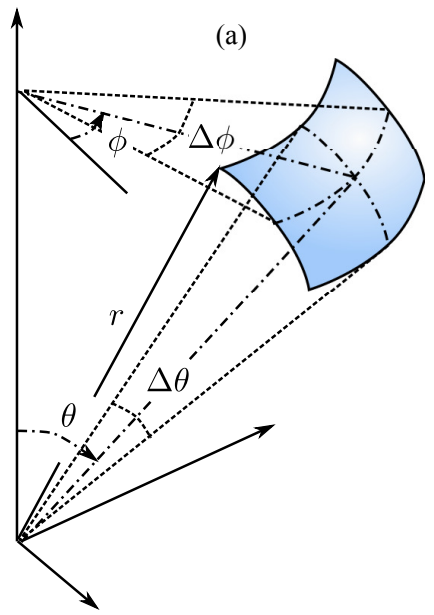

(b)

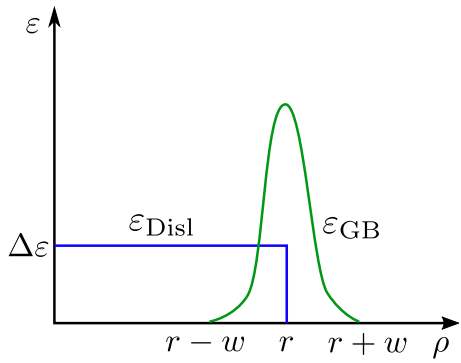

(c)

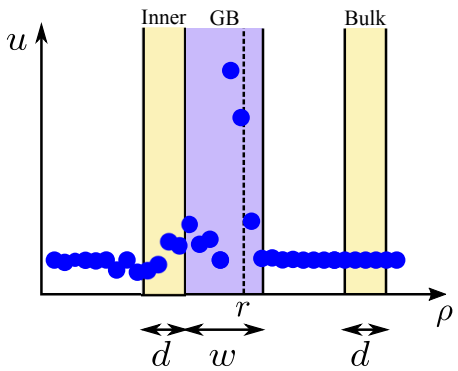

Figure 2. An approach to analysing the contributions to grain boundary driving force on a local and atomistic basis. (a) the definition of a grain boundary surface element; (b) contributions to the excess energy of atoms as a function of distance from the centre of the grain; (c) a schematic representation of the regions used to calculate contributions to the driving force. See main text for definition of regions and labels.

value of strain, the majority of dislocations are of $1 / 6\langle 112\rangle$ (Shockley) and $1 / 6\langle 110\rangle$ (Stair-rod) type, with the former increasing and the latter decreasing in fraction with increasing strain. Note that these dislocation densities are several orders of magnitude larger than those found in cold-worked metal Humphreys and Hatherly 2004].

\begin{tabular}{|c|c|c|c|c|c|c|}
\hline \multirow[t]{2}{*}{ Strain } & \multirow{2}{*}{$\begin{array}{c}\text { Energy } \\
\text { density } \\
(\mathrm{meV} / \text { atom })\end{array}$} & \multirow{2}{*}{$\begin{array}{c}\text { Effective } \\
\text { pressure } \\
(\mathrm{GPa})\end{array}$} & \multirow{2}{*}{$\begin{array}{c}\text { Dislocation } \\
\text { density } \\
\left(10^{17} \mathrm{~m}^{-2}\right)\end{array}$} & \multicolumn{3}{|c|}{$\begin{array}{c}\text { Fraction of dislocations } \\
\text { by burgers vector }(\%)\end{array}$} \\
\hline & & & & $\begin{array}{l}1 / 2\langle 110\rangle \\
\text { (Perfect) }\end{array}$ & $\begin{array}{l}1 / 6\langle 112\rangle \\
\text { (Shockley) }\end{array}$ & $\begin{array}{l}1 / 6\langle 110\rangle \\
\text { (Stair-rod) }\end{array}$ \\
\hline $20 \%$ & 7.75 & 0.28 & 0.346 & 0.9 & 21.1 & 76.9 \\
\hline $60 \%$ & 11.9 & 0.43 & 1.803 & 3.9 & 47.4 & 45.8 \\
\hline $80 \%$ & 11.0 & 0.39 & 1.638 & 4.8 & 58.4 & 33.5 \\
\hline $90 \%$ & 13.7 & 0.49 & 2.421 & 5.2 & 56.0 & 35.2 \\
\hline $95 \%$ & 14.9 & 0.54 & 3.135 & 5.2 & 59.4 & 31.0 \\
\hline $98 \%$ & 26.0 & 0.93 & 3.388 & 12.9 & 52.8 & 5.0 \\
\hline
\end{tabular}

Table 1. Excess energy density and dislocation content in deformed material as a function of applied uniaxial strain.

The case of $98 \%$ is an exception to the patterns seen in the other cases. A large fraction of the dislocations identifiable in the final state are of no particular type. Furthermore, as we shall see, the resulting grain boundary migration behaviour does not fit with the pattern shown by the other strains. We can attribute this anomalous behaviour to a small-size effect in our simulations. Our simulation cell is sufficiently small that the initial uniaxial strain of $98 \%$ crushes the material down to a layer only $5 \AA$ thick. The crystal lattice reorients under the deformation such that 


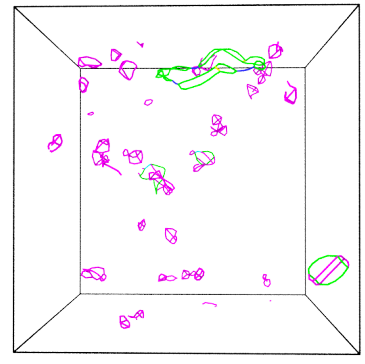

(a) $20 \%$

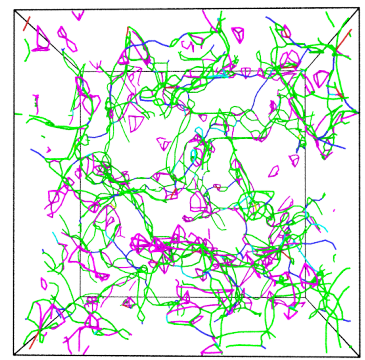

(d) $90 \%$

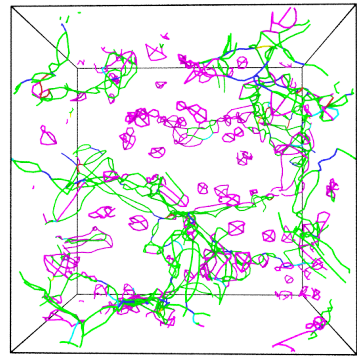

(b) $60 \%$

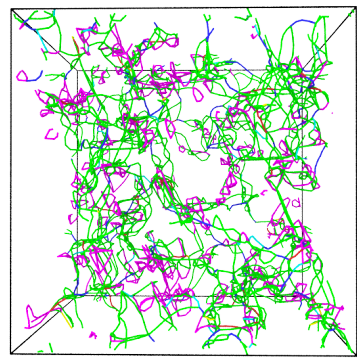

(e) $95 \%$

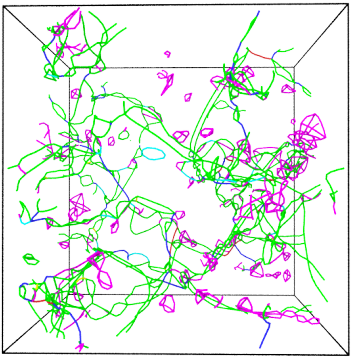

(c) $80 \%$

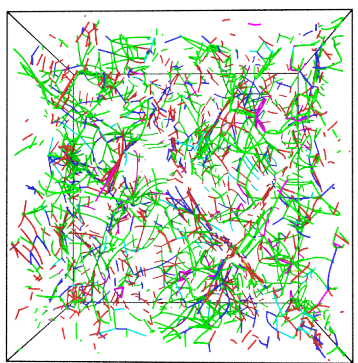

(f) $98 \%$

Figure 3. Visualisations of the dislocation networks generated by plastic strain, analysed using the Ovito software Stukowski 2010. The dislocation types shown, by burgers vector, are $1 / 2\langle 110\rangle$ (blue), $1 / 6\langle 112\rangle$ (green), and $1 / 6\langle 110\rangle$ (magenta). The dislocations shown in red in (f) are of no particular type.

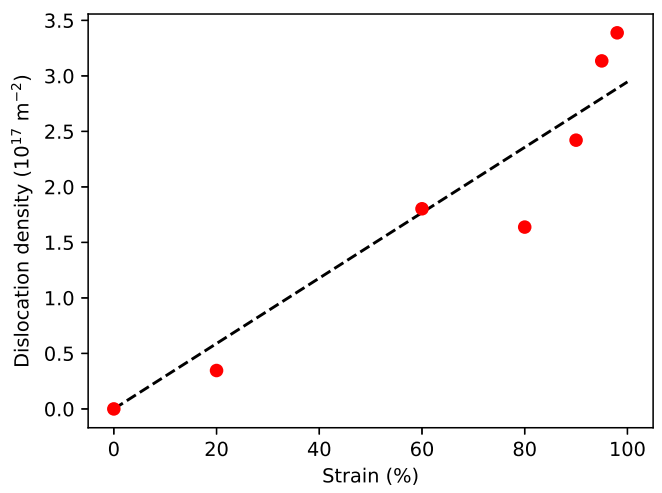

Figure 4. Dislocation line density in deformed material as a function of applied strain. The dashed line shows a best-fit straight line through the origin. 
the $\langle 111\rangle$ poles lie parallel to the strain axis and the material breaks up into many nanoscale grains. Furthermore, the thin slab accommodates only three close-packed layers of atoms and so the periodic cell becomes quasi-two-dimensional, restricting the pattern of dislocations that can form to include only line segments lying parallel to the strain axis. On applying the biaxial strain to return the cell to a cubic geometry, the polycrystalline nature of the material and the reorientation of the lattice persist, such that the cell illustrated in Figure 3(f) contains a nano-crystalline sample with a high density of high-angle grain boundaries.

\subsection{Migration simulations}

We now present the results of some preliminary simulations demonstrating the usefulness of the approach described in Section 2. We consider two sets of simulations to explore the effect on grain boundary migration of the degree of plastic deformation in the central grain and the angle of misorientation between the central grain and the matrix.

3.2.1. Effect of strain To explore the effect of plastic strain we take spheres of material from the deformation simulations at seven values of the plastic strain between $0 \%$ and $98 \%$ and insert these into a cubic grain of defect-free material with a misorientation of $36.87^{\circ}$ about the [001] axis, corresponding to a $\Sigma 5$ misorientation. Snapshots of the evolving simulation cell are taken periodically, the atomic configurations are relaxed and our analysis process as described in Section 2.3 is applied. Figure 5 shows the two contributions to the driving force for grain boundary migration averaged over the surface of the spherical grain. The capillary force is by far the dominant contribution for an inclusion of this size and we can see that this contribution is approximately constant over the set of simulations, as we would expect. The defect contribution rises with increasing plastic strain, but remains significantly smaller. Note that the case of $98 \%$ strain is anomalous, as we discussed above.

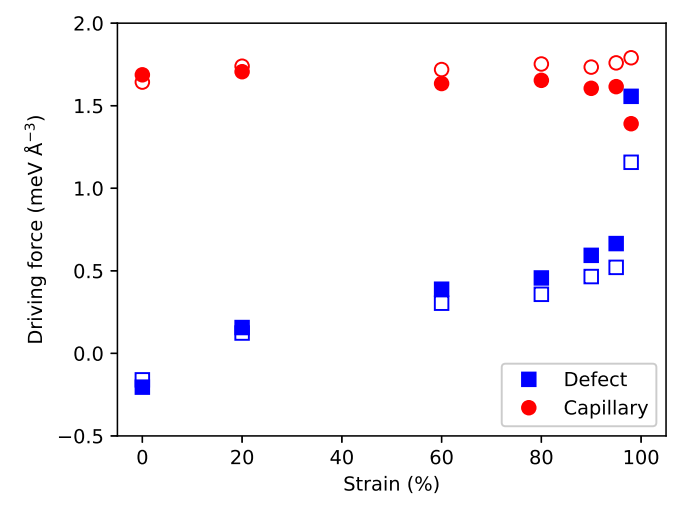

Figure 5. Contributions to the driving force for grain boundary migration as a function of the plastic strain applied to the central, shrinking grain. The filled and open symbols show respectively the forces including and prior to inclusion of the correction term $\Delta_{P}$ discussed in Section 2.3

Figure 6 shows the time evolution of the average radius of the shrinking grain for 


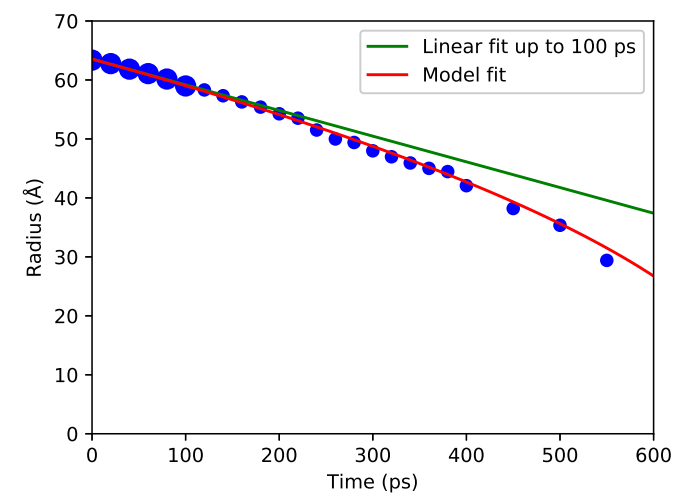

(a)

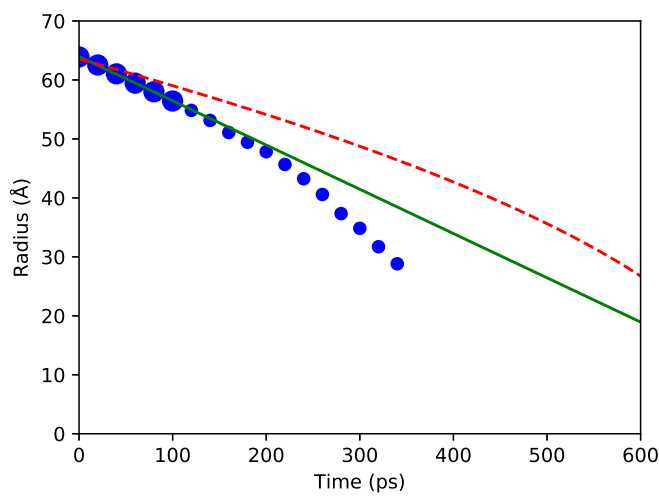

(b)

Figure 6. The trajectories for the average radius of the central shrinking grain in the case of (a) $0 \%$ and (b) $95 \%$ plastic strain. The green lines show a linear fit to the initial shrinkage velocity over $100 \mathrm{ps}$. The red lines show a parabolic shrinkage profile with an initial shrinkage rate fitted to the $0 \%$ strain trajectory (solid in (a), reproduced dashed in (b), see main text for discussion).

the case of (a) $0.0 \%$ strain and (b) $95 \%$ strain. The initial shrinkage velocities are indicated for comparison and in the $0 \%$ strain case we show the parabolic trajectory obtained by assuming that the initial rate of shrinkage, $\mathrm{d} r /\left.\mathrm{d} t\right|_{0}$ is driven solely by the capillary force due to the excess grain boundary energy, extracting this grain boundary energy, $\gamma_{\text {eff }}$, from the initial shrinkage rate, $\mathrm{d} r /\left.\mathrm{d} t\right|_{0}=-2\langle m\rangle \gamma_{\text {eff }} / r_{0}$, and assuming that the grain boundary energy remains constant over the course of the simulation so

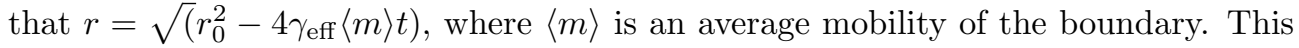
parabolic trajectory is reproduced on the data for the $95 \%$ strain simulation and the increase in the shrinkage rate due to the plastic deformation defects can clearly be seen. We note that in all cases the central grains are observed to shrink to zero size within the simulation timescale, but that detection and fitting of the grain surface becomes challenging for a grain with a radius below $\sim 25 \AA$.

We next examine how well the variation with plastic strain in the initial shrinkage velocity as measured in the simulations conforms to our expectations based on our analysis of the contributions to the grain boundary driving force over the surface of the shrinking grain. We would expect that the initial shrinkage velocity would be

$$
\begin{aligned}
\left.\frac{\mathrm{d} r}{\mathrm{~d} t}\right|_{0} & =-\langle m\rangle\left(P_{\text {Disl }}+P_{\mathrm{GB}}\right) \\
& =-\langle m\rangle\left(\left[P_{\text {Inner }}+\Delta_{P}\right]+\left[P_{\text {Interface }}-\Delta_{P}\right]\right),
\end{aligned}
$$

where $\langle m\rangle$ denotes an average boundary mobility over the surface of the central grain. We can use the values of the driving forces calculated from the relaxed simulation snapshots (and shown in Figure 5) and a value for $\langle m\rangle$ calculated from the parabolic shrinkage of the $0 \%$ strain case (Figure 6(a)) to obtain predictions of the initial shrinkage velocities. These predictions are compared to the initial rates of shrinkage measured from the simulation trajectories (examples of which are shown in Fig 6) as a function of strain in Figure 7. Whilst the agreement between the predictions and the measured rates of shrinkage is far from perfect, the overall trend is reasonably well captured. Recalling that the estimated contributions to the driving forces used 


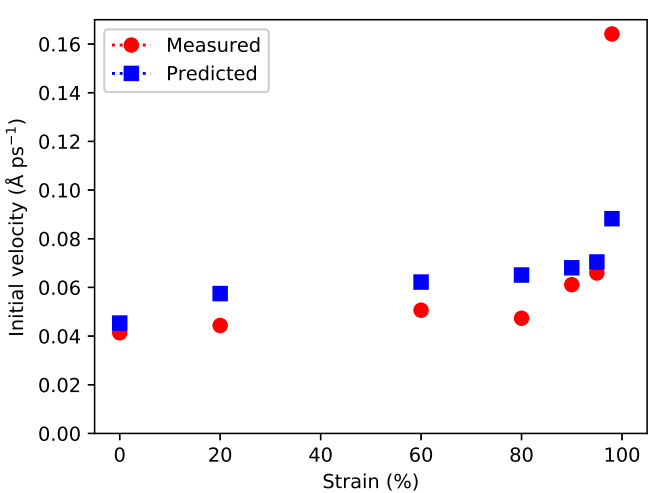

(a)

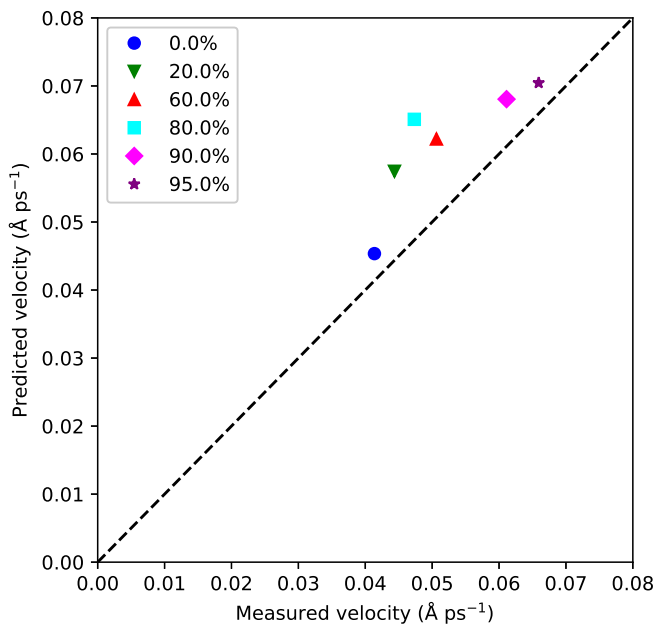

(b)

Figure 7. A comparison of the initial rates of shrinkage determined from the simulation trajectories as a function of plastic strain with predictions based on an atomistic analysis of driving forces outlined in Section 2.3 (a) velocities as a function of plastic strain, and (b) a direct comparison of predicted and measured velocities (98\% strain case omitted).

in the predictions are based purely on the relaxed potential energies of individual atoms in the vicinity of the interface, then it may be unreasonable to expect closer agreement than this. Certainly the role of the deformation defects in enhancing the rate of shrinkage of the central grain is strongly indicated by the results in Figure 7 . We note that, once again, the $98 \%$ strain case is an anomaly due to the nature of the deformed microstructure.

3.2.2. Effect of misorientation angle We now consider the results of a second series of simulations in which we vary the degree of misorientation, still about the [001] axis, between the deformed grain and the matrix, from 6 to 45 degrees. In this misorientation series we include three "special" misorientations: the $\Sigma 13\left(22.62^{\circ}\right)$, $\Sigma 17\left(28.07^{\circ}\right)$ and $\Sigma 5\left(36.87^{\circ}\right)$. For each misorientation we have performed a pair of simulations, one each with a central grain deformed to either $0 \%$ or $95 \%$ strain.

Figure 8 shows the two contributions derived from an atomistic analysis, as outlined in Section 2.3, to the driving force for grain boundary migration. The contribution from plastic deformation defects is largely independent of the misorientation angle, as it should be if our approach to analysis is correct. In contrast, the capillary force due to the excess energy of the grain boundary itself shows a strong variation with misorientation angle. Several aspects of the variation are of interest.

First we note that there is a systematic trend of increasing capillary force with increasing angle at lower angles. We expect this relationship between misorientation angle and grain boundary energy, but the systematic trend persists up to $>20^{\circ}$, which is beyond the usual transition from low-angle to high-angle type boundary behaviour Stukowski 2010.

Second, we also note that the "special", low- $\Sigma$ boundaries do not have particularly 


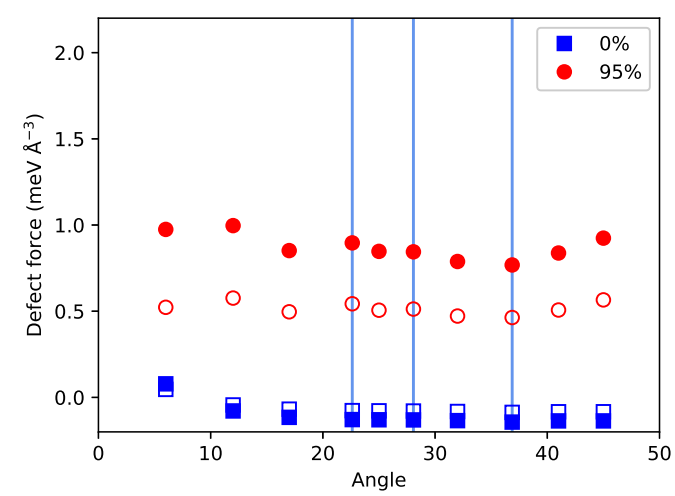

(a)

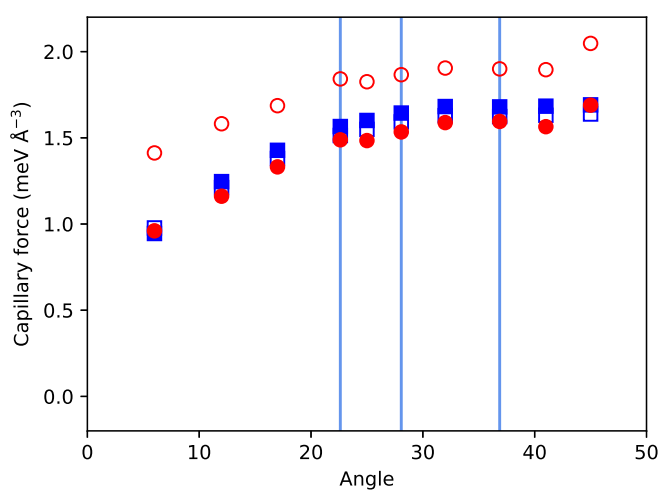

(b)

Figure 8. The driving forces for grain boundary migration due to (a) plastic deformation defects and (b) the grain boundary, as a function of misorientation angle for zero and $95 \%$ plastic strain. In each case the filled and open symbols show the forces including and prior to inclusion of the correction term $\Delta_{P}$.

low grain boundary energy. This is most likely a result of both the small size of the inclusion and the fact that all possible boundary plane normals are represented in our system and the variation in grain boundary energy with plane normal is at least as strong as that with misorientation angle Olmsted et al. 2009a b.

Finally, we can see from Figure 8 that the correction term $\Delta_{P}$ is particularly important in this case. Only by applying the correction do we obtain a series of values for the capillary force which is independent of the plastic strain in the inclusion. Without the correction, a portion of the defect energy is misallocated as a contribution to the grain boundary energy, giving an unphysical picture of the driving forces at work.

In the introduction we raised the possibility that a significant effect in deformation driven boundary migration might be a 'washing out' of anisotropy in the behaviour. We now examine our results for any sign of such effects, noting from the outset the limited nature of the data that we have at our disposal here. Figures 9(a) and (b) show a measure of the anisotropy of the grain boundary energy as the grain boundary normal varies over the surface of the spherical grain. We see the variation in the mean, standard deviation and maximum and minimum grain boundary energies as a function of angle for the zero and $95 \%$ strain cases. In both cases, one standard deviation of variation is around $15 \%$ of the absolute grain boundary energy, which is a similar degree of variation to that of the mean energy with misorientation angle, as we might expect. In Figure 9(c) we compare the ratio of the standard deviation in boundary energy to the mean boundary energy for each misorientation angle. This measure of anisotropy is plotted for the $95 \%$ against the $0 \%$ strain case and we can see that it is systematically lower in the presence of plastic deformation.

We can also look for anisotropy in the shape of the shrinking, initially spherical, grains. Figure 10 (a) shows the shape of the central grains after $20 \mathrm{ps}$ of evolution for the first six misorientation angles for the strained and unstrained cases. We can see that for the unstrained case, in which boundary motion is driven only by the capilliary force, there is a tendency for the central grain to become somewhat oblate. 


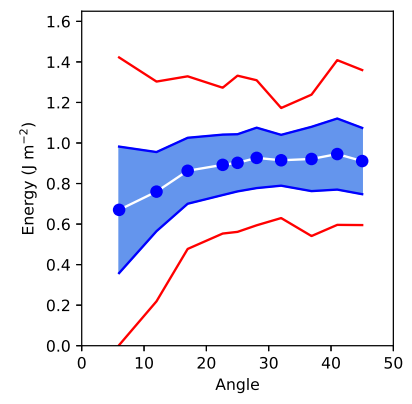

(a)

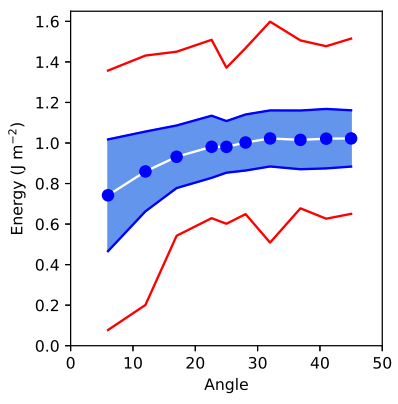

(b)

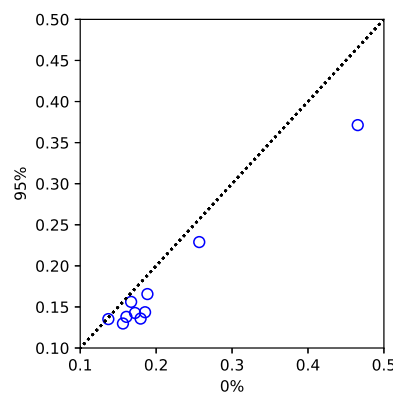

(c)

Figure 9. Anisotropy in interfacial energy as a function of misorientation angle. (a) and (b) show the mean (circles), standard deviation (blue bands) and min/max (red lines) of the grain boundary energy over the surface of the shrinking grain. (c) shows the ratio of the standard deviation to the mean for the $0 \%$ and $95 \%$ strain cases for each misorientation angle as an indication of the effect of plastic strain on the degree of anisotropy.

This tendency reduces with increasing misorientation angle and disappears above $28^{\circ}$. In contrast, the strained central grain maintains a more spherical shape over the full range of misorientation angles. The exception to these trends is the lowest angle case of a $6^{\circ}$ misorientation in which the strained grain is somewhat prolate.

Figure 10(b) shows the variation of a measure of the shape anisotropy as a function of misorientation angle. This measure is calculated from a list of the radii $\left\{r_{i}\right\}$ and polar angles $\left\{\theta_{i}\right\}$ of the sections over the grain surface as

$$
\mathcal{S}=\sum_{i} 2 \pi w\left(\theta_{i}\right) \Delta \theta \sin \left(\theta_{i}\right) r_{i} / n_{i}
$$

where we apply a weight as a function of $\theta$ defined as follows:

$$
\begin{array}{rll}
w(\theta)=1 & \text { if } & |\theta-\pi / 2| \leq \pi / 6 \\
-1 & \text { if } & |\theta-\pi / 2|>\pi / 6 .
\end{array}
$$

This leads to a measure of anisotropy $\mathcal{S}$ that is positive when the grain is oblate and negative when it is prolate. The value of $\mathcal{S}$ plotted in Figure 10(b) is normalised such that it will be unity for an oblate grain with a polar diameter equal to half the equatorial diameter.

\subsection{Origin of driving forces}

Our approach to analysing the contributions to the driving force for grain boundary migration is fundamentally atomistic, calculating excess energies as sums over individual atom energies in regions in and around the grain boundary. This is a necessary approach where the driving forces are expected to vary strongly with position on the boundary surface. However, the intuitive physical picture that we have of these contributions to the driving force relies on the idea of extended defects: the capillary force arises due to a tendency to minimise the area of the grain boundary (a twodimensional defect) and the additional recrystallisation driving force arises due to the presence of a network of dislocations (one-dimensional defects). In the following 


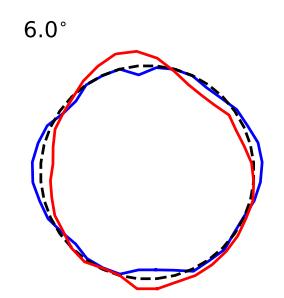

$22.62^{\circ}$
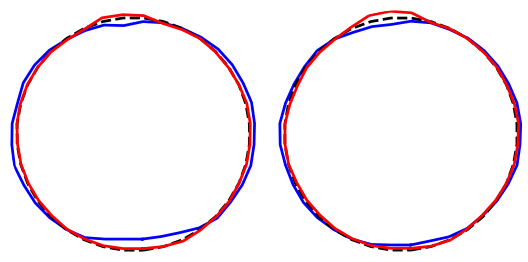

(a)

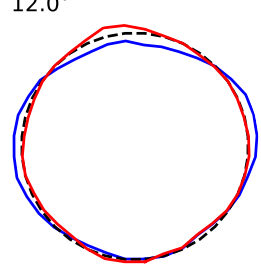

$25.0^{\circ}$

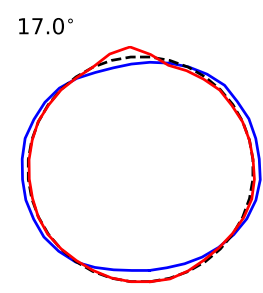

$28.07^{\circ}$

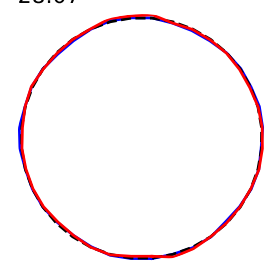

.

Figure 10. Anisotropy in grain shape as a function of misorientation angle. (a) shows the shape of the shrinking grain after 20 ps for the $0 \%$ (blue) and $95 \%$ (red) cases for the first six misorientation angles. The polar angle $\theta$ varies from zero at the top of each image to $\pi$ at the bottom. For each value of $\theta$ the outline of the shape is an average over the azimuthal angle from zero to $\pi$ on the right and from $\pi$ to $2 \pi$ on the left of the shape. (b) shows a measure of the shape anisotropy (defined in Section 3.2.2 as a function of misorientation angle for the strained and unstrained cases.

sections we will compare our atomistically derived measure of the driving force with an analysis of the defect content of the simulation cells.

3.3.1. Interfacial energy and capillary force The local interfacial energy is calculated as the excess potential energy, over perfect bulk crystal, of the atoms lying in a solid angle element and in a small thickness either side of the nominal grain boundary position. Maps of this measure of grain boundary energy are shown in Figures 11.(a) and (c) corresponding to misorientation angles of $6.0^{\circ}$ and $28.07^{\circ}$ respectively for a central grain with zero plastic strain. In both cases, the energy varies significantly over the surface, dropping almost to zero in places in the case of the $6.0^{\circ}$ misorientation. In Figure 11(a) we have marked the positions of a selection of crystallographic poles in the outer grain, most of which correspond to regions of low energy in the map. We can compare the positions of these poles with the network of intrinsic grain boundary dislocations making up the interface in the inset Figure 11(b). It can be clearly seen that the poles lying at low energy points in the map are also removed from the positions of dislocations. The exception to this is the $\langle 100\rangle$-type pole visible in the figures which lies directly on a structural dislocation and is correspondingly at a position of high local energy in the map. The atomistically-based measure of grain boundary energy is thus consistent with a defect-based view of the interface.

In Figure 11(c), the map of the energy of the $28.07^{\circ}$ boundary, the poles are again marked. In addition, points of particularly high energy are indicated by black dots. These black dots are reproduced on the inset Figure 11(d) along with the dislocation network associated with the grain boundary $\$$. We can clearly see that these points

$\S$ It is of note that this grain boundary network can be identified even though a $28.07^{\circ}$ misorientation 


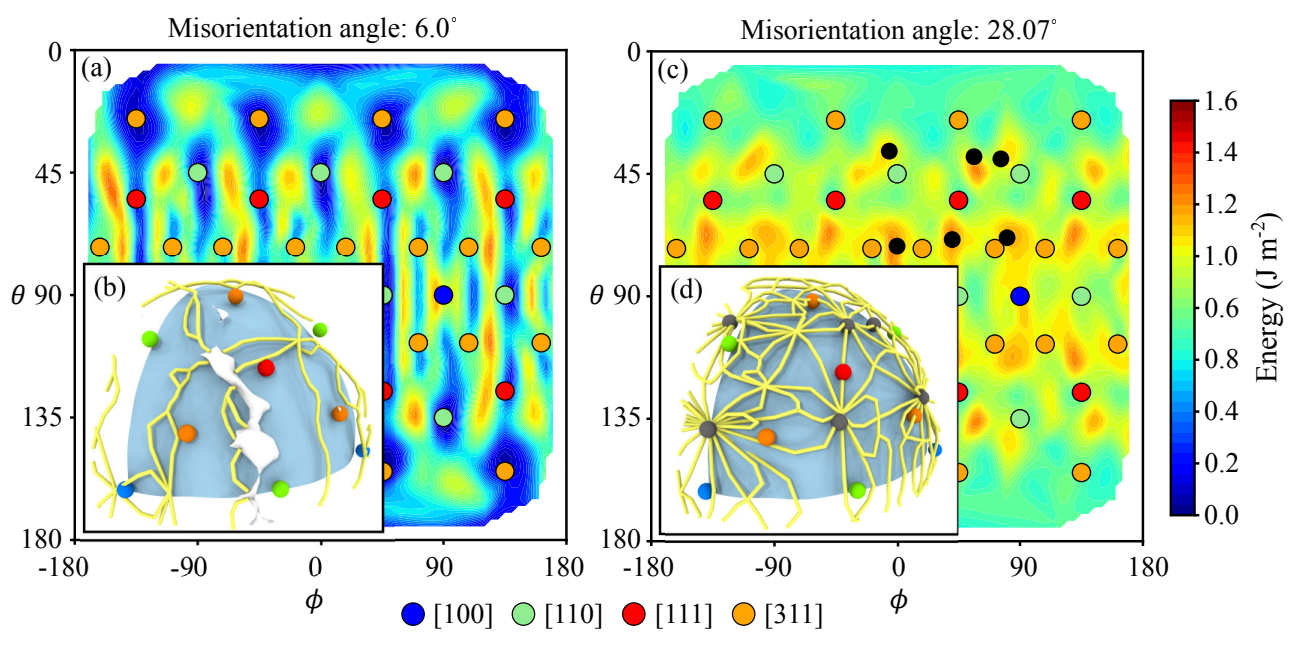

Figure 11. (a) and (c) show maps of the interfacial (grain boundary) excess energy density over the surface of the spherical grain for two misorientation angles. Crystallographic poles of interest are marked by coloured dots. The black dots in (c) indicate regions of high energy on the surface. The inset figures (b) and (d) show the dislocation network (as identified by the Ovito software Stukowski 2010]) within the grain boundary region with the same set of crystallographic poles marked. The light grey surfaces in the insets are included in order to make the dislocation network clearer.

of high energy correspond to the nodes of the grain boundary dislocation network and the atomistic measure of energy is again rationalised in terms of the pattern of extended defects.

3.3.2. Dislocations and defect force We next consider the driving force due to plastic deformation defects within the central grain. Figures 12(a) and (c) show maps of this driving force over the full angular range for the $6.0^{\circ}$ and $28.07^{\circ}$ misorientations with a central grain made up of material strained to $95 \%$. Again, significant angular variation is evident. Black circles mark locations of particularly high driving force in Figure 12(a). Since this component of the driving force is due to deformation in the central grain, the positions of these high-force points should move systematically as the misorientation angle is changed (since we have used the same sphere of material in all the cells). Replotting these locations in Figure 12 (c) for the $28.07^{\circ}$ misorientation angle with a shift in azimuthal angle of $(28.07-6.0=22.07)^{\circ}$ shows that this is indeed the case. By examining the network of deformation dislocations inside the spherical grain, shown in Figures 12(b) and (d), we can confirm that these highdriving-force locations correspond to points at which the dislocation network enters the grain boundary region.

The artificially constructed systems used in molecular dynamics simulations can often contain features additional to those intended by their creators and it is important

angle places this boundary in the high-angle range. For the slightly larger $32.0^{\circ}$ misorientation no network can be identified. Furthermore, the grain boundary networks in both the $6.0^{\circ}$ and the $28.07^{\circ}$ misorientation boundaries remain identifiable when the central grain is made up of material strained to the $95 \%$ level. 

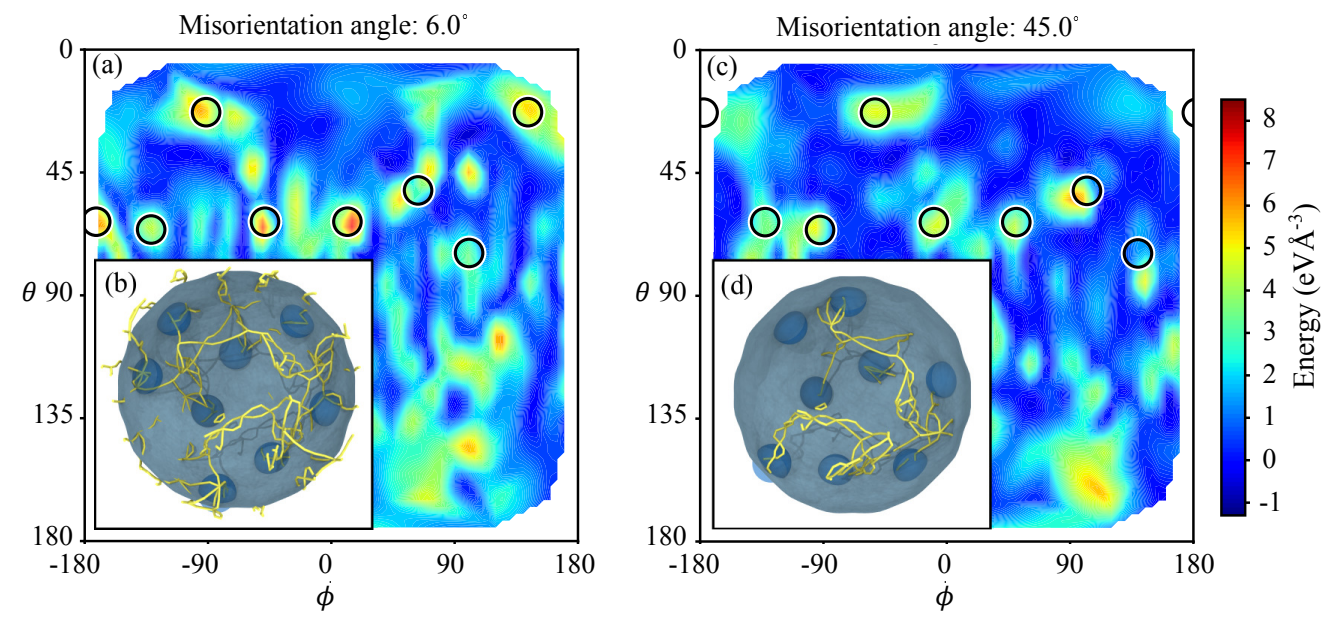

Figure 12. (a) and (c) show maps of the driving force for grain boundary migration due to plastic deformation within the central grain resolved over the whole spherical surface. The black circles indicate regions of high energy density in (a). These black circles are again shown in (c) in positions rotated by $22.07^{\circ}$ (corresponding to the difference in misorientation between (a) and (c)) and can be seen to again correspond to regions of high energy. The inset figures (b) and (d) show the dislocation network (as identified by the Ovito software Stukowski 2010 ) in the interior of the spherical grain. The dark grey hemispheres correspond to the positions marked by the dots in (a) and (c). The light grey surfaces in the insets lie just within the nominal grain boundary and are included in order to obscure the dislocation network due to the grain boundary itself.

to look for the consequences of the unexpected. In our case, it is possible that the cutting and insertion of a spherical inclusion could lead to an increase in the energy density of the central grain over that of the surrounding bulk. For example, the network of dislocations might lead to a different average lattice constant and so an excess hydrostatic pressure on the shrinking grain. To rule out such effects we have looked at the distribution of individual atomic potential energies of the inner and outer grains and confirmed that the excess energy of atoms in the inner grain is indeed localised around the dislocations. Atoms further from the dislocations do not have a potential energy significantly different to those in the matrix grain.

\subsection{Migration mechanisms}

Though it is beyond the scope of the present study to examine the mechanisms of boundary motion in detail, we show in Figure 13 that portions of the grain boundary surface that are intersected by dislocations in the defect network within the deformed grain show a tendency to move inwards slightly ahead of the surrounding boundary. A similar bowing out of the moving grain boundary near dislocations was observed in the bicrystal simulations of Godiksen et al Godiksen et al. 2007a This may be due to the additional driving force for motion provided by the dislocations at these points or it may be due to the dislocations providing focal points for the operation of a faster mode of grain boundary migration than that operating in regions away 
from the dislocation network. Experimental observations have shown a correlation between the local rate of grain boundary migration during recystallisation and the stored energy density in the vicinity of the boundary (see, for example, the work of Zhang et al. Zhang et al. [2014]). The labelled points in Figures 13(b) and (c) show a case where the intersection of a dislocation line shifts from A to B over time. The boundary at A remains relatively advanced even after the dislocation line moves away, suggesting that this effect is not a reversible deformation of the boundary due to interaction with the dislocation. After it has moved to B, this dislocation line causes the boundary at this point to advance also.
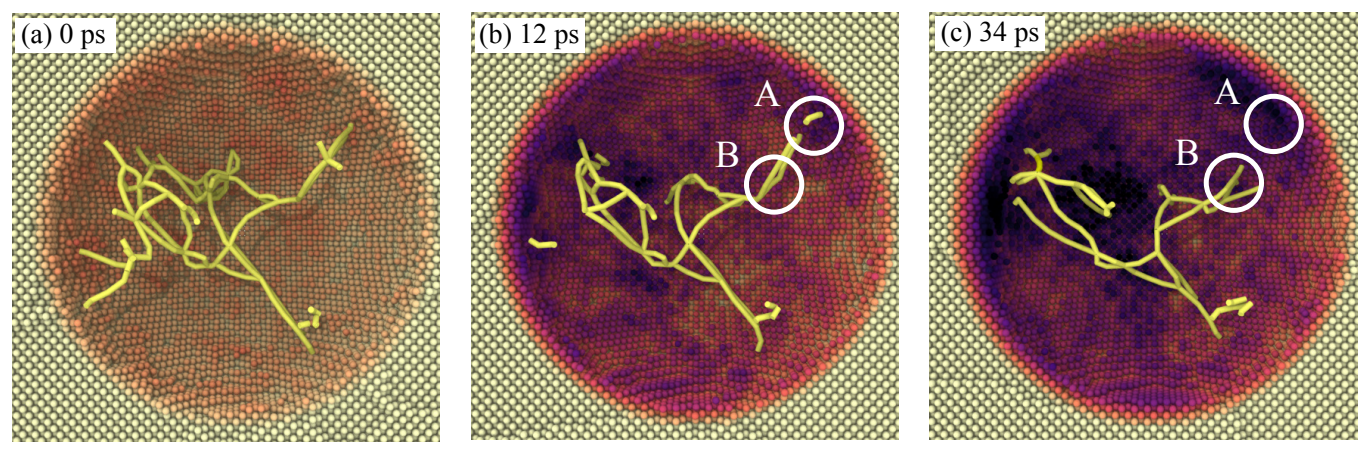

Figure 13. Snapshots of the shrinking grain, deformed to $95 \%$ and misaligned by $6^{\circ}$ about [100] with the surrounding grain. Atoms with the local environment of the shrinking grain are invisible. Those in the matrix grain are coloured according to their distance from the centre of the grain such that atoms in regions where boundary motion has progressed further inwards are darker. The grain boundary network due to plastic strain is shown by the yellow lines. Points A and B are discussed in Section 3.4

\section{Conclusions}

We have demonstrated that molecular dynamics can be used to simulate the migration of grain boundaries driven by the presence of a complex network of dislocations arising from plastic deformation and that we can analyse and rationalise the behaviour at the atomistic level. In particular we have shown:

- We can set up simulations in which grain shrinkage is (in part) driven by plastic deformation damage;

- We can use the individual atomic potential energies to calculate the individual contributions to the driving force and map them over the surface of the shrinking grain;

- The shrinkage rate of the central grain grain behaves as expected according to a simple physical model and the calculated driving force contributions;

- The interfacial energy shows a somewhat reduced anisotropy (variation with boundary plane) in the presence of plastic deformation. We also observe a reduced anisotropy in the shape of the shrinking grain;

- The migration of the boundary appears to be focused on areas where the dislocation network intersects with the grain boundary. 
Several areas for further attention stand out. First, the results presented here consider only the earliest stages of grain shrinkage, during which the grain remains approximately spherical. Longer simulations would be illuminating, in particular looking at the changing shape of the shrinking grain and the effect of plastic deformation on the anisotropy. Second, simulations with a larger central grain, in which the defect-based driving force is a more significant contribution would more strongly reveal the effects we aim to study. Both longer and larger simulations are entirely plausible. Third, we have barely begun to analyse the mechanism of boundary motion, when driven by dislocation defects. Careful study of the regions identified in Figure 13 will provide insight into the role of plastic deformation in, for example, providing a focal point for the local atomic rearrangement that takes place during grain boundary migration. Other areas for investigation include simulations with a lower, more realistic, dislocation density and with the dislocations arranged in a more typical way, simulations with a differently shaped, e.g. elongated, central grain and simulations of a shrinking grain in a matrix of multiple grains.

\section{Acknowledgments}

C.P.R. was funded by a University Research Fellowship of The Royal Society. Simulations were carried out on the University of Manchester's Computational Shared Facility. Research data supporting this publication and Jupyter Notebooks incorporating our analysis and the plots of results used in the figures are available online Race 2019.

\section{Appendix}

\section{References}

\section{References}

Fadi Abdeljawad, Stephen M. Foiles, Alexander P. Moore, Adam R. Hinkle, Christopher M. Barr, Nathan M. Heckman, Khalid Hattar, and Brad L. Boyce. The role of the interface stiffness tensor on grain boundary dynamics. Acta Materialia, 158:440-453, 2018. Link. URL Link.

J. H. Driver, D. Juul Jensen, and N. Hansen. Large strain deformation structures in aluminium crystals with rolling texture orientations. Acta Metallurgica et Materialia, 42(9):3105-3114, 1994. Link. URL Link.

R. B. Godiksen, Z. T. Trautt, M. Upmanyu, S. Schmidt, and D. Juul Jensen. Towards atomic level simulations of recrystallisation -setting up suitable geometry. Materials Science $\mathscr{E}$ Technology, 21(12):1373-1375, 12 2005. URL Link.

R B N Godiksen, S Schmidt, and D Juul Jensen. Molecular dynamics simulations of grain boundary migration during recrystallization employing tilt and twist dislocation boundaries to provide the driving pressure. Modelling and Simulation in Materials Science and Engineering, 16(6):065002, 2008. URL Link.

Rasmus B. Godiksen, Zachary T. Trautt, Moneesh Upmanyu, Jakob Schitz, Dorte Juul Jensen, and Sren Schmidt. Simulations of boundary migration during recrystallization using molecular dynamics. Acta Materialia, 55(18):6383 - 6391, 2007a. ISSN 1359-6454. Link. URL Link. 
Rasmus B. Godiksen, Zachary T. Trautt, Moneesh Upmanyu, Søren Schmidt, and Dorte Juul Jensen. Simulation of recrystallization using molecular dynamics; effects of the interatomic potential. Materials Science Forum, 558-559:1081-1086, 2007b. Link.

G. Gottstein and L.S. Shvindlerman. Grain Boundary Migration in Metals Thermodynamics, Kinetics, Applications. CRC Press, 2010.

$\mathrm{E} \mathrm{R}$ Homer. Investigating the mechanisms of grain boundary migration during recrystallization using molecular dynamics. IOP Conference Series: Materials Science and Engineering, 89(1):012006, 2015. URL Link.

F.J. Humphreys and M. Hatherly. Introduction. In F.J. Humphreys and M. Hatherly, editors, Recrystallization and Related Annealing Phenomena (Second Edition), pages 1 - 10. Elsevier, Oxford, second edition edition, 2004. ISBN 978-0-08-0441641. Link, URL Link.

Koenraad G. F. Janssens, David Olmsted, Elizabeth A. Holm, Stephen M. Foiles, Steven J. Plimpton, and Peter M. Derlet. Computing the mobility of grain boundaries. Nat Mater, 5(2):124-127, 02 2006. URL Link.

Manping Liu, Hans J. Roven, Yingda Yu, and Jens C. Werenskiold. Deformation structures in 6082 aluminium alloy after severe plastic deformation by equal-channel angular pressing. Materials Science and Engineering: A, 483-484:59-63, 2008. Link. URL Link,

Mark A. Miodownik. A review of microstructural computer models used to simulate grain growth and recrystallisation in aluminium alloys. Journal of Light Metals, 2 (3):125-135, 8 2002. URL Link.

David L. Olmsted, Stephen M. Foiles, and Elizabeth A. Holm. Survey of computed grain boundary properties in face-centered cubic metals: I. grain boundary energy. Acta Materialia, 57(13):3694 - 3703, 2009a. ISSN 1359-6454. Link. URL Link.

David L. Olmsted, Elizabeth A. Holm, and Stephen M. Foiles. Survey of computed grain boundary properties in face-centered cubic metals-ii: Grain boundary mobility. Acta Materialia, 57(13):3704 - 3713, 2009b. ISSN 1359-6454. Link. URL Link.

Steve Plimpton. Fast parallel algorithms for short-range molecular dynamics. Journal of Computational Physics, 117(1):1-19, 3 1995. URL Link.

C. P. Race. Data for paper: Atomistic simulations of grain boundary migration under recrystallisation conditions, May 2019. URL Link.

C. P. Race, J. von Pezold, and J. Neugebauer. Role of the mesoscale in migration kinetics of flat grain boundaries. Physical Review B, 89(21):214110, June 2014. Link. URL Link.

C. P. Race, R. Hadian, J. von Pezold, B. Grabowski, and J. Neugebauer. Mechanisms and kinetics of the migration of grain boundaries containing extended defects. Physical Review B, 92(17):174115-, 11 2015. URL Link.

Alexander Stukowski. Visualization and analysis of atomistic simulation data with ovito-the open visualization tool. Modelling and Simulation in Materials Science and Engineering, 18(1):015012, 2010. URL Link.

A. P. Sutton and R. W. Balluffi. Interfaces in crystalline materials. Oxford: Clarendon Press, 1995. 
Luis A. Zepeda-Ruiz, Alexander Stukowski, Tomas Oppelstrup, and Vasily V. Bulatov. Probing the limits of metal plasticity with molecular dynamics simulations. Nature, 550:492 EP -, 09 2017. URL Link.

Yubin Zhang, Andy Godfrey, and Dorte Juul Jensen. In-situ investigation of local boundary migration during recrystallization. Metallurgical and Materials Transactions A, 45(6):2899-2905, 2014. Link. URL Link.

Rajendra R. Zope and Y. Mishin. Interatomic potentials for atomistic simulations of the ti-al system. Phys. Rev. B, 68(2):024102, Jul 2003. Link. 